Student Research Club of Cardiology at the Department of Cardiology and Internal Medicine, Nicolaus Copernicus University in Torun, Ludwik Rydygier Collegium Medicum in Bydgoszcz

\title{
Does SARS-CoV-2 infect cardiomyocytes directly? Yes, it does
}

\section{Corresponding author:}

Anna Ryszewska, Student Research Club of Cardiology at the Department of Cardiology and Internal Medicine, Nicolaus Copernicus University in Torun, Ludwik Rydygier Collegium Medicum in Bydgoszcz, Jagiellonska 13/15, 85-067 Bydgoszcz, Poland, e-mail: a.ryszewska@onet.pl

Medical Research Journal 2021; Volume 6, Number 3, 237-244 10.5603/MRJ.a2021.0038 Copyright @ 2021 Via Medica ISSN 2451-2591 e-ISSN 2451-4101

\begin{abstract}
Introduction: COVID-19 (Coronavirus disease 2019) appeared in Wuhan, China, at the ending of 2019. The SARS-CoV-2 virus which causes the illness has spread all over the world and caused a pandemic. The first target of the virus is the respiratory tract; however, the COVID-19 may present different types of course. It is known that the SARS-CoV-2 affects multiple organs, including the heart. Cardiac manifestations of COVID-19 include myocarditis, myocardial infarction, heart failure, acute coronary syndrome, arrhythmia. The authors know about the patients who had only cardiovascular complications due to the COVID-19. Several mechanisms of heart injury are considered and so is the direct infection. Aim of the study: The present review aimed to find out if the SARS-CoV-2 may infect the heart directly and in which mechanism. The review is an information collection considering the SARS-CoV-2 impact on the heart. Material and methods: The authors have made research using the PubMed search engine to find studies and case reports considering the cardiovascular implications of COVID-19. The signs and symptoms in patients with cardiac implications were studied. The authors have also checked if studies explaining does the SARS-CoV-2 affects the heart directly were conducted.

Results: SARS-CoV-2 brings several cardiovascular signs such as changes in imaging tests and elevation of several laboratory markers. The changes may suggest myocarditis or mimic cardiac infarction. The SARS-CoV-2 may affect cardiomyocytes indirectly by causing hypoxia and cytokine storm. As the heart tissue presents a high level of ACE2 which is the target of the virus, the SARS-CoV may infect cardiomyocytes directly. The hypothesis was confirmed in endomyocardial biopsies, autopsy, and in vitro studies.

Conclusions: The SARS-CoV-2 impacts several organs. The heart may be injured indirectly (hypoxia and cytokine storm) and directly (ACE2 present in the heart), which gives consequences in a clinical course. The direct injury was confirmed in a variety of ways.

Key words: SARS-CoV-2, COVID-19, cardiomyocyte
\end{abstract}

Med Res J 2021; 6 (3): 237-244

\section{Introduction}

The history of coronavirus disease 2019 (COVID-19) has begun in Wuhan in China at the ending of 2019 where inhabitant doctors encountered pneumonia of unknown aetiology. On 8 January 2020 , the first information that the novel coronavirus could be responsible for this unknown pneumonia appeared [1]. Two days later, the genome sequence of severe acute respiratory syndrome coronavirus 2 (SARS-CoV-2) was obtained [2]. The World Health Organization declared a pandemic on 11 March 2020 [3]. Since the beginning of the pandemic, SARS-CoV-2 has spread all over the world. To date, there have been $160,040,871$ confirmed cases of COVID-19, including 3,4494,758 deaths, reported to WHO [4]. The COVID-19 has varied clinical courses which can be divided into asymptomatic infection, mild upper respiratory tract illness, moderate upper respiratory tract illness, severe upper respiratory tract illness, and even death. The severity of clinical manifestation is associated with risk factors, such as gender, age whether the presence of comorbidities like diabetes, cardiovascular disease, and immunological disorders [5]. The main sign due to SARS-CoV-2 infection concerns the 
respiratory tract, however, cardiovascular involvement has been noticed in several studies. Cardiac manifestations of COVID-19 include myocarditis, myocardial infarction, heart failure, acute coronary syndrome, arrhythmia. The previous coronavirus epidemic has also shown cardiovascular complications as hypotension, arrhythmia, and sudden cardiac death. Influenza virus affects the heart similarly to SARS-CoV-2 as well [6]. Furthermore, COVID-19 may cause renal dysfunction, gastrointestinal complications, liver dysfunction, neurological and haematological disorders, and vascular abnormalities. Extrapulmonary symptoms have been reported in previous coronavirus epidemics, which have been associated with increased mortality [7]. In this review, the authors describe cardiovascular involvements of SARS-CoV-2 infection, their possible pathogenesis, and signs and try to prove that SARS-CoV-2 can affect cardiomyocytes directly.

\section{Materials and methods}

A literature search was conducted using PubMed to identify relevant studies. Keywords used during searching are in Table 1. The date of publication was between 01.01.2020 and 19.05.2021, the texts written in English. The accepted studies included original research, review, and letters.

The primary inclusion criteria were autopsy, biopsy, and in vitro studies regarding the presence of viruses in heart tissue. Review articles were excluded. Review articles were used to write the introduction and propose mechanisms of myocardial damage during COVID-19. If the abstract did not contain any information about research virus genome into cardiomyocytes the articles were excluded as well.

To write the introduction the PubMed database was searched using "SARS-CoV-2" or "COVID-19", and "Cardiomyocytes" keywords. Subsequently, the most interesting articles in the authors' opinion were chosen.

\section{Discussion}

Viral infections are known to affect cardiac functions. The heart is the second target of SARS-CoV-2 after the lungs [8]. Nevertheless, the impact of Sars-CoV-2 has not been widely described yet.

During the Sars-CoV-2 pandemic, differential diagnosis of cardiovascular diseases (CVD) should include COVID-19 even in patients without typical symptoms of COVID-19 such as fever or cough [9]. Clinicians have known several signs of cardiac injury caused by SARS-CoV-2.

In several cases the electrocardiography test showed ST-segment elevation in lateral (I, aVL) leads [9-12], ST-segment depression in aVR [9, 10], T-wave inversion [10], low QRS voltage [9, 10, 12] and sinus tachycardia $[8,10]$. The important fact is that these patients did not have a history of heart diseases [8, $10,12]$. Patients with CVD presented similar findings in ECG $[9,11]$ Moreover, new arrhythmias appeared in the COVID-positive patients [13, 14]. SARS-CoV-2 is proven to lead to arrhythmias significantly more frequently than other coronaviruses [15]. Diffuse elevation of ST-segment may appear in patients with myocarditis [16] which is a possible cause of cardiac injury connected with COVID-19 [17]. However, ST-segment elevations should be interpreted carefully because they may mimic a STEMI [11]. Cardiac arrhythmias such as AV block, VF, and VT are risk factors of death in patients with myocarditis [8].

One of the most valuable tests in describing COVID-19 impact on the heart is echocardiography. Features include severely depressed left ventricular ejection volume (LVEF) - about 30-40\% [8-10, 12], hypokinesis of the left ventricle, heart apex [9-11] and right ventricle [9], enlarged left ventricle [8, 9], pulmonary hypertension [8], increased wall thickness [9, 10, $18]$ and pericardial effusion $[10,18]$. The findings were not present in previous echocardiograms [11] and disappeared after recovery $[9,10,12]$

Table 1. Keywords used during the searching

\begin{tabular}{llll}
\hline Virus & COVID-19 & Heart & Intervention \\
- SARS-CoV-2 & • COVID-19 & - Heart & - Endomyocardial biopsy \\
- 2019-nCoV & - SARS-CoV-2 Infection & - Cardiomyocyte & - Cardiosphere \\
- Severe acute respiratory & - 2019-nCoV Infection & - Myocarditis & - Heart slice \\
syndrome Coronavirus 2 & • 2019-nCoV Disease & - Endocarditis & - Engineered heart tissues \\
- SARS Coronavirus 2 & - Pneumonia & - Pericarditis & - Autopsy \\
- SARS virus & & - Heart failure & - Echocardiography \\
- Coronavirus & & - Cardiac failure & - Histopathology \\
& & - Cardiogenic shock & \\
& & - Myocardial Ischemia & \\
& & - Myocardial infarction & \\
& & - ACE-2 & \\
& &
\end{tabular}


Table 2. Information about original studies

\begin{tabular}{lcc}
\hline Authors & Type of study & Methods used in studies \\
\hline L. Bailey et al. [27] & Original research article & In vitro \\
D. Bojkova et al. [28] & Original research article & In vitro \\
A. Sharma et al. [29] & Original research article & In vitro \\
P. Wenzel et al. [30] & Research letter & Endomyocardial biopsy \\
F. Escher et al. [31] & Original research article & Endomyocardial biopsy \\
G. Tavazzi et al. [32] & Case report & Endomyocardial biopsy \\
Z. Varga et al [33] & Research letter & Autopsy \\
D. Lindner et al. [34] & Original research article & Autopsy \\
\hline M. Pesaresi et al. [35] & Original research article & Autopsy \\
B. T. Bradley et al. [36] & Original research article & Autopsy \\
G. Pietro Bulfamante et al. [37] & Original research article & Autopsy
\end{tabular}

Table 3. TnT level impacts several laboratory parameters [13] which is shown in the table below

\begin{tabular}{lcc}
\hline HDL & Elevated TnT levels & Normal TnT levels \\
LDL & & Did not differ \\
Triglyceride & Did not differ & \\
CRP & higher & Lower \\
Procalcitonin & Significantly higher & Lower \\
globulin & Significantly higher & Lower \\
CK-MB test & Significantly higher & Lower \\
Myoglobin & Significantly higher & Lower \\
NT-proBNP & Significantly higher & Lower \\
Lactic acid & higher & Lower \\
Respiratory dysfunction & higher & Lower \\
Creatinine & More severe & Mild \\
AST & higher & Lower \\
ALT & higher & Lower
\end{tabular}

ALT — alanine aminotransferase; AST — aspartate aminotransferase; CK-MB test — creatine kinase-myocardial band test; CRP — C-reactive protein; HDL — high-density lipoprotein; LDL — low-density lipoprotein; NT-proBNP — N-terminal pro-brain natriuretic peptide; TnT — troponin T

Magnetic resonance imaging (MRI) may be used to confirm myocarditis in patients with COVID-19 $[11,18]$. Lake Louise Criteria include injury of myocardium on T1-weighted image with late gadolinium enhancement and myocardial oedema on T2-mapping sequences [17]. It may present biventricular myocardial oedema, biventricular hypokinesis, increased thickness of the wall, and decreased LVEF $[10,18]$. MRI is an alternative test to myocardial biopsy [12].

Laboratory findings are important factors of heartinjury. High-sensitivity troponin T (TnT) was often increased in patients with COVID-19 [10-13]. Myocardial injury, which is associated with a severe course of COVID-19, may lead to cardiac dysfunction and arrhythmias. TnT level above the $99^{\text {th }}$ percentile confirms the diagnosis [19]. In the case series study conducted by T. Guo et al. [13], the myocardial injury appeared in $27.8 \%$ of patients with COVID-19. The scientists confirmed the relationship between TnT level in patients with myocardial injury and mortality. Out of patients without underlying cardiovascular diseases (CVD) died 7.62\% with normal TnT levels and $37.5 \%$ with elevated TnTs. The highest mortality was in patients with elevated TnTs and underlying CVD (69.44\%). In comparison to patients without TnT elevation, increased TnT level was associated with more frequent complications. The authors indicated possible 
risk factors of TnT elevation - i.e. older age, men sex, comorbidities such as hypertension, cardiomyopathy, coronary disease, chronic obstructive pulmonary disease, chronic kidney disease [13].

Another predictive factor is the N-terminal pro-brain natriuretic peptide (NT-proBNP) [13] which may be used as a myocardial injury marker [8]. Elevated levels of NT-proBNP were observed in several patients with COVID-19-induced myocarditis $[8,10,11,13]$.

Speed of TnT and NT-proBNP concentration changes is also significant. Dynamic elevation appeared in patients who ultimately died while gradual changes were evident in those who survived. [13]

$D$-dimer may be elevated in patients with COVID-19 and be associated with multiorgan dysfunction [12]. A Higher D-dimer level is associated with a fatal course of COVID-19 and is taken as a death risk factor [20]. Scientists hypothesize that elevated D-dimer may be a result of microvascular thrombosis connected with inflammation [12]. Elevation of D-dimer was seen in several patients with cardiovascular complications of COVID-19 [9, 11-14]. A possible mechanism of heart injury connected with thrombosis is acute coronary syndrome [18].

Interleukin-6 is an inflammatory cytokine that marks cytokine storm $[12,18]$, as previously mentioned. Interleukin-6 levels were elevated in several cases $[8,9,11$, $12,18]$ as well as C-reactive protein and ferritin which also appear in cytokine-induced cardiac injury $[12,18]$. Moreover, the elevation of Interleukin- 6 and ferritin levels were connected with a higher risk of death [20].

SARS-CoV-2, as well as SARS-CoV, infects cells thanks to the ability to bind to angiotensin-converting enzyme 2 (ACE2) [21]. SARS-CoV-2 similarly to other coronaviruses has 4 structural proteins (spike-S, envelope-E, membrane-M, nucleocapsid-N). Protein S acts the main role in infecting cells. When coronaviruses enter the target cell the protein $\mathrm{S}$ is cleaved at $\mathrm{S} 1$ and S2 sites. The fusion of viral and cellular membrane is possible thanks to subunit S2, which binds with ACE2 and it causes virus endocytosis. Protein $S$ is divided by a cellular protease such as Transmembrane protease serine 2 (TMPRSS2), Cathepsin B (CTSB), Cathepsin L (CTSL), but only TMPRSS2 activity is necessary for viral spread and pathogenicity whereas cathepsin B/L activity is dispensable [22, 23]. ACE2 is present in every part of the human body. The heart is one of many organs with a high level of ACE2 expression. SARS-Cov-2 has a much bigger affinity to ACE2 than SARS. In one autopsy study, scientists discovered copies of the SARS-CoV-2 from 16 of 22 patients who died due to COVID-19. In the second study, a high viral load was detected among 39 people who died due to COVID-19. On the other hand, $38 \%$ of these patients had not SARS-CoV-2 in the heart. Such myocardial injury, reflected by an increased level of troponin is a risk factor for in-hospital mortality. The concentration of troponin correlates with mortality. Also, patients with an elevated level of troponin demonstrate a higher concentration of inflammatory markers. Myocarditis is a severe complication of COVID-19. Unlike SARS, SARS-CoV-2 creates a lymphocyte inflammatory response. Several cases of myocarditis imitate a heart attack. Except for direct impact on myocardiocytes, SARS-CoV-2 may affect endothelial cells [24]. SARS-CoV-2 attachment to ACE2 may lead to a change of ACE2 signalling pathway causing lung and heart injury [25]. During SARS-CoV-2 infection, the serum level of inflammatory mediators increases, such as C-reactive protein (CRP), ferritin, IL-6, IL-2, TNF, IL-10, lactic dehydrogenase (LDH). All of them may cause cytokine storms. The highest concentration of these markers occurs in severe upper respiratory tract illness and is related to immune response and inflammatory reactions which are dynamic [5].

Immune reactions can be divided into 3 phases: early infection, a pulmonary phase, and a severe hyper inflammation phase. Most patients do not develop a severe hyper inflammation phase.

Unfortunately, several per cent of sick people get into the most severe phase. During this phase, the human immune response develops cytokine storm which may injure organs different than lungs [26]. The respiratory tract is the main beneficiary of SARS-CoV-2 infections. Infection and immune response are responsible for pulmonary tissue injury, endothelial permeability, vasodilatation, and leukocyte recruitment which lead to lung damage and hypoxemia. Ten per cent of patients with lung damage and hypoxemia develop acute respiratory distress syndrome (ARDS) [6]. According to what is written several mechanisms responsible for the cardiac injury can be identified.

1. Indirect myocardial injury:

a. Severe hypoxia caused by lung damage and acute respiratory distress syndrome - hypoxia is responsible for cardiac injury;

b. Cytokine storm caused by the huge immune response - immune cells and immune proteins damage cardiomyocytes.

2. Direct myocardial injury caused by direct cardiomyocytes infection by SARS-CoV-2 - the heart has a high level of ACE2 expression making the heart a target for the virus.

\section{Results}

A. L. Bailey et al. [27] tested samples derived from 4 patients suffering from COVID-19 and myocarditis. These have been obtained thanks to autopsy and 


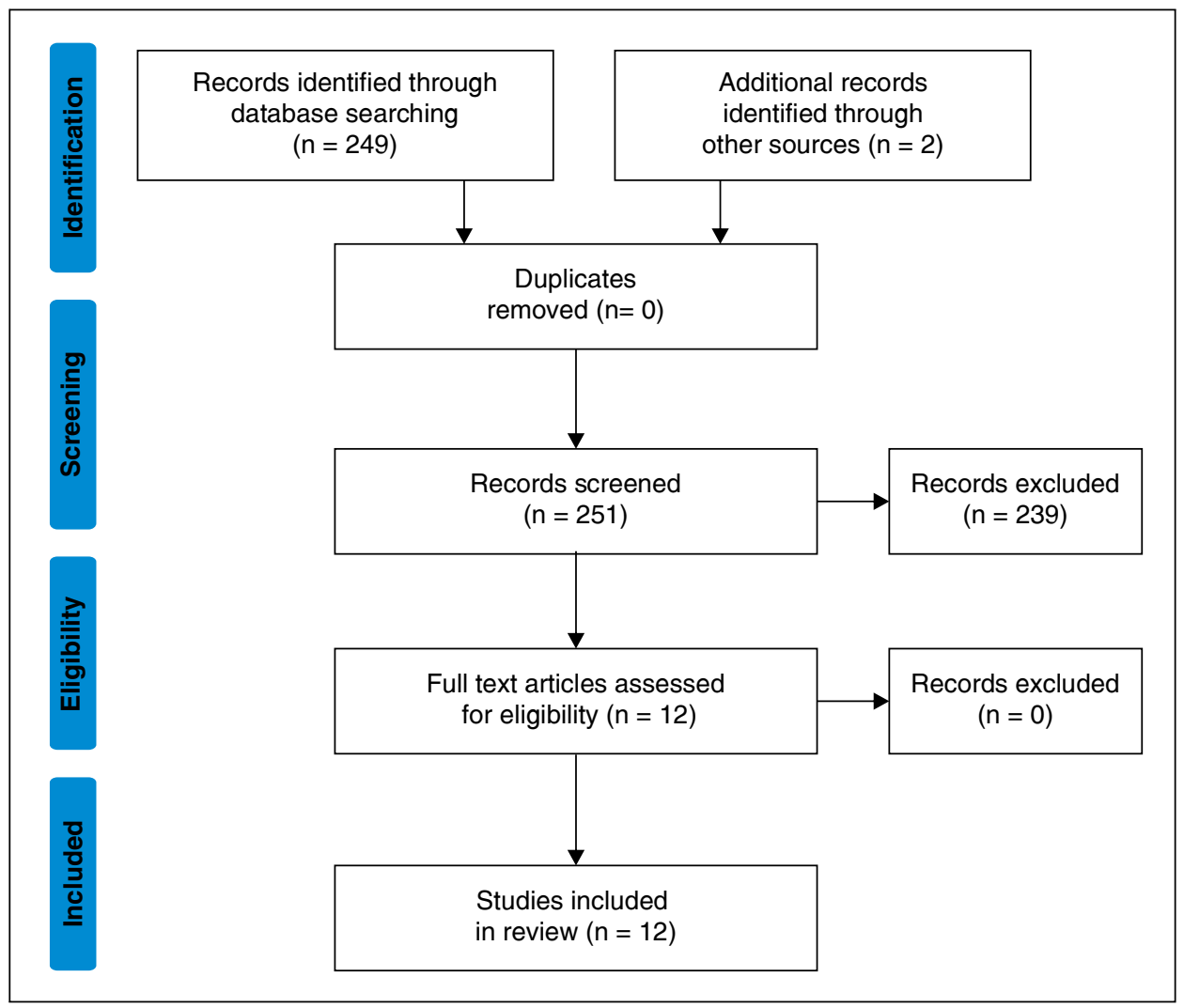

Figure 1. Flow diagram

endomyocardial biopsy. Coronary angiography was normal, with no signs of luminal stenosis or thrombosis what was confirmed in the autopsy. SARS-CoV-2 infection was detected by polymerase chain reaction (PCR) from nasopharyngeal samples. In postmortem microscopic examination of the left ventricle images of necrosis, degenerative vacuolization of cardiomyocyte cytoplasm, and mononuclear cell infiltration were obtained. Negative controls were autopsied heart samples from patients with metastases and neurodegenerative diseases. The researchers detected SARS-CoV-2 spike and nucleocapsid RNA in the samples of myocardium in every patient with COVID-19-related myocarditis. Viral transcripts were mainly detected within cells that were morphologically compatible with cardiomyocytes. These transcripts were also detected in lung airway epithelial cells, rarely in myocardial pericytes and adipocytes. The transcripts were located in cytoplasmic and perinuclear locations. The scientists checked the susceptibility of different myocardial cells to SARS-CoV-2 and if the virus can develop in myocardium bred combinations of fibroblasts, macrophages, and human-induced pluripotent stem cell-derived cardiomyocytes (hiPSC-CMs) with the virus. Only combinations with hiPSC-CMs cardiomyocytes showed the production of viruses and viral RNA. The researchers proved also that numerous genes in different cells were regulated upon SARS-CoV-2 infec- tion. What is interesting, the cells which did not show viral replication also had changed gene expression. It may mean that the SARS-CoV-2 can change gene expression without direct viral infection. Overall, genes responsible for immune cell activation were upregulated and genes related to metabolism, mitochondrial function, muscle contraction showed downregulations. The scientists used engineered heart tissue (EHT) in their research. They wanted to check if EHTs mimics aspects of COVID-19 myocarditis. The examinations showed that SARS-CoV-2 infected EHTs showed a reduced speed of contraction and relaxation. This may be caused by left ventricular dysfunction reported during COVID-19-related myocarditis. Many mechanisms may be responsible for the decline in cardiac contractility, including cell death, sarcomere structure, immune responses, metabolism dysfunction. The researchers confirmed ACE2 mRNA expression in cardiac tissue.

D. Bojkova et al. [28] also cultured cells with SARS-CoV-2. The results show that the virus can undergo a complete replication cycle in human-induced pluripotent stem cell-derived cardiomyocytes (hiPS-CMs). Moreover, the conducted laboratory tests showed the activation of various signalling pathways, including interferon. 3D tissue demonstrated signs of tissue injury. The virus was found in cardiomyocytes using electron microscopy. The researchers performed an endomyo- 
cardial biopsy in one patient with COVID-19 myocarditis symptoms and detected the virus by electron microscopy as well. The scientists also confirmed ACE2 mRNA expression in heart tissue. What is interesting, the mRNA expression was not present in undifferentiated iPS cells and ACE2 expression in cardiomyocytes was lower and localized more to perinuclear and cytoplasmic regions as against other TMPRSS2-positive cells.

Conclusions which are provided by research by A. Sharma et al. [29] are that SARS-CoV-2 may invade hiPSC-CMs in vitro. The virus enters the cells via ACE2. Cytopathic effect and replication of the virus trigger apoptosis of cardiomyocytes. hiPSCs were extracted from peripheral blood mononuclear cells and differentiated into cardiomyocytes. The differentiation was confirmed by levels of cardiac troponin T and -actinin. The hiPSC-CMs were exposed to SARS-CoV-2 infection for $72 \mathrm{~h}$. The results in infected cells were compared to the mock cells. Infected cells were positive for viral spike protein - it means that SARS-CoV-2 is can cardiomyocytes directly. The cytopathic effect occurred after $72 \mathrm{~h}$ and was verified by cleaved caspase-3, which is an apoptosis marker, and by the double-stranded DNA (dsDNA). The infected cells were positive for both which suggests virus-induced apoptosis. After $72 \mathrm{~h}$ of infection, the hiPSC-CMs discontinued beating, possibly because of cell-cell gap junctions disruption. What is interesting, previous treatment of infected cells with an ACE-2 antibody reduced the level of cleaved caspase-3 and inhibited apoptosis. However, the virus was still able to infect the cells - it may suggest variant ways of internalization.

P. Wenzel et al. [30] took an endomyocardial biopsy from two patients with COVID-19-related myocarditis symptoms and who were admitted to the hospital. Both patients had signs of COVID-19 up to 4 weeks before the biopsy. SARS-CoV-2, influenza A and B, metapneumovirus, parainfluenza virus, respiratory syncytial virus were excluded using laboratory tests on the day of admission. Pathomorphological tests revealed myocardial inflammation with the presence of lymphocytes (mainly cytotoxic T cells) and macrophages without cardiomyocyte necrosis. Furthermore, the real-time polymerase chain reaction (RT-PCR) assay confirmed the presence of the SARS-CoV-2 genome in biopsy samples. Lower or negative viral load for CMV, EBV, adenovirus, parvovirus B19, HHV6, and Coxsackie virus were also detected. During hospitalization, IgG antibodies for SARS-CoV-2 were detected.

F. Escher et al. [31] obtained endomyocardial biopsies of 104 patients. Every patient had angiography to exclude coronary artery disease. Most of the patients were men (76\%), mean age was $57.9 \pm 16,4$, the diagnosis: active myocarditis $13.4 \%$, inflammatory cardiomyopathy $32.6 \%$, borderline myocarditis $2.9 \%$, dilated cardiomyopathy $41.3 \%$, amyloidosis $9.6 \%$. SARS-CoV-2 genome was detected in the myocardial biopsy in 5 of 104 patients (RT-PCR). Parvovirus B19 genome was detected in 70 of 104 patients, HHV6 genome in 8 patients, EBV in 4 patients, and Coxsackie virus in 1 patient. In SARS-CoV-2 positive samples, only parvovirus B19 was detectable. Histological images of SARS-CoV-2-positive samples showed: 1 active myocarditis (according to the Dallas criteria), 1 borderline myocarditis (according to the Dallas criteria), and 3 inflammatory cardiomyopathies. An inflammatory infiltration consisting of macrophages, T lymphocytes, and memory T cells (CD45R0) was found in 4 of 5 SARS-CoV-2 positive samples. All of these 5 patients had elevated numbers of cell adhesion molecules (CD54/ICAM-1).

The case report performed by G. Tavazzi et al. [32] has proved myocardial localization of the SARS-CoV-2. Scientists have described a case of a 69-year-old patient who suffered from COVID-19. The clinical manifestation suggested severe acute myocarditis. An endomyocardial biopsy was performed. The biopsy demonstrated CD68-positive macrophages with damaged membrane, particles of the SARS-CoV-2 virus in interstitial cells which lost cytoplasmatic membrane integrity; and low-grade myocardial inflammation. Viral particles were not observed in endothelia and myocytes, however, cardiomyocytes showed focal lysis of myofibrils and lipid droplets. The presence of macrophages may suggest the migration of infected macrophages from the respiratory tract to extra-pulmonary tissues. The case report may be taken as evidence of SARS-CoV-2-connected direct heart damage and also damage associated with the infected macrophages migration in patients with COVID-19 presenting clinical manifestation of acute myocarditis.

Z. Varga et al. [33] proved that SARS-CoV-2 can infect endothelial cells and cause endothelial inflammation as well. The findings revealed the presence of SARS-CoV-2 elements within endothelial cells, inflammatory infiltration, apoptosis, and pyroptosis. COVID-19 endotheliitis may be responsible for the systemic impaired microcirculatory function.

D. Lindner et al. [34] have conducted an autopsy study that included patients fatal because of COVID-19 (confirmed post mortem by RT-PCR). 39 patients in a median age of 85 were included in the study. Almost $90 \%$ (35 of 39) of them died because of pneumonia. The cause of death of the rest of the patients was unknown. Myocarditis wasn't documented among the group (according to the Dallas criteria). Some of the patients suffered from comorbidities, including coronary artery disease, hypertension, and diabetes. 2-4 days after death, samples of cardiac tissue were collected. Scientists performed quantitative 
RT-PCR for SARS-CoV-2. For paraffin sections in situ hybridization was conducted. SARS-CoV-2 was found in $61.5 \%$ (24 of 39 ) patients: in the 8 cases virus load was below 1000 copies per microgram RNA and in the 16 cases virus load was more than 1000 copies. In 5 patients who had the highest virus load, replication of SARS-CoV-2 was detected. In situ hybridization showed the localization of SARS-CoV-2 - interstitial cells and macrophages invading the myocardium.

M. Pesaresi et al. [35] have used Transmission Electron Microscopy (TEM) and Scanning Electron Microscopy to identify the SARS-CoV-2 particles in samples from 3 organs: heart, lung, and kidney. The samples were collected from 2 dead patients with COVID-19 (confirmed post mortem by RT-PCR). In one of the cases, viral particles were present in the cardiomyocytes adjoining myofibrils. The finding was observed in TEM and then confirmed by SEM analysis. TEM showed dissimilar morphology of the viral particles, which may suggest a different state of viral replication or tissue damage. Moreover, the SARS-COV-2 virus was also identified in the heart using RT-PCR.

B.T. Bradley et al. [36] have conducted a post-mortem examination on 14 patients who died with COVID-19. All patients suffered from comorbidities and were in the median age of 73.5 years. Half of the patients were examined by in-situ dissection and the rest of them by standard autopsy procedure. The scientists used light and electron microscopy, immunohistochemistry, and quantitative RT-PCR. SARS-CoV-2 RNA was detected in cardiac tissue in 2 patients. In one of them, lymphocytic myocarditis was detected. The sample of myocardial tissue taken from the patient was positive for SARS-CoV-2 RNA by PCR, however, immunohistochemistry and electron microscopy did not confirm the finding. The scientists made a hypothesis that the presence of viral RNA in several extra-pulmonary tissues may be a result of contamination by the virus which is circulating.

G. Pietro Bulfamante et al. [37] conducted 6 autopsies -5 males and 1 female with COVID-19. The mean age was 59.5. Every patient died of respiratory failure due to COVID-19 and did not show clinical signs of left ventricle damage. PCR assay of heart tissue samples confirmed the presence of the SARS-CoV-2 genome. The viral genome was found in lung extracts as well. The tests were negative in healthy control. In the light microscopy, researchers confirmed the presence of SARS-CoV-2 nucleoprotein and spike protein. The viral proteins were discovered within cardiomyocytes and in cytosolic areas of lipofuscin. Thanks to the RNAScope assay, scientists were able to determine, if the SARS-CoV-2 is transcriptionally active in cardiomyocytes. The test proved that the virus can replicate in cardiomyocytes. In this study, in contrast to the study by Z. Varga et al., it was not shown endothelial cytopathic effects nor endotheliitis. Electron microscopy corroborated evidence on the presence of SARS-CoV-2 in cardiomyocytes.

\section{Conclusion}

The study limitation is that one cannot exclude an important role for hypoxemia, thrombosis, and inflammation. The research material was not big, these studies were performed on a small number of samples. Moreover, the COVID-19 pandemic does not last long. The longer it lasts, the bigger number of research will be conducted. Also, long-term effects are probable to be seen further in the future.

Fortunately, the studies were of high quality, which gives greater confidence that the pieces of evidence are authentic. Cited examples prove that SARS-CoV-2 may affect cardiomyocytes directly.

\section{Conflict of interest: None.}

\section{Funding: None.}

\section{References}

1. Bogoch II, Watts A, Thomas-Bachli A, et al. Pneumonia of unknown aetiology in Wuhan, China: potential for international spread via commercial air travel. J Travel Med. 2020; 27(2), doi: 10.1093/jtm/taaa008, indexed in Pubmed: 31943059.

2. Zheng J. SARS-CoV-2: an emerging coronavirus that causes a global threat. Int J Biol Sci. 2020; 16(10): 1678-1685, doi: 10.7150/ijbs.45053, indexed in Pubmed: 32226285.

3. WHO Director-General's opening remarks at the media briefing on COVID-19 - 11 March 2020. https://www.who.int/director-general/speeches/detail/who-director-general-s-opening-remarks-at-the-media-briefing-on-covid-19---11-march-2020 (10.04.2021).

4. WHO Coronavirus (COVID-19) Dashboard. https://covid19.who.int/ (27.05.2021).

5. García LF. Immune response, inflammation, and the clinical spectrum of COVID-19. Front Immunol. 2020; 11: 1441, doi: 10.3389/fimmu.2020.01441, indexed in Pubmed: 32612615.

6. Goha A, Mezue K, Edwards P, et al. COVID-19 and the heart: An update for clinicians. Clin Cardiol. 2020; 43(11): 1216-1222, doi: 10.1002/clc.23406, indexed in Pubmed: 32533585

7. Behzad S, Aghaghazvini L, Radmard AR, et al. Extrapulmonary manifestations of COVID-19: Radiologic and clinical overview. Clin Imaging. 2020; 66: 35-41, doi: 10.1016/j.clinimag.2020.05.013, indexed in Pubmed: 32425338

8. Zeng JH, Liu YX, Yuan J, et al. First case of COVID-19 complicated with fulminant myocarditis: a case report and insights. Infection. 2020; 48(5): 773-777, doi: 10.1007/s15010-020-01424-5

9. Fried J, Ramasubbu K, Bhatt R, et al. The Variety of cardiovascular presentations of COVID-19. Circulation. 2020; 141(23): 1930-1936, doi: 10.1161/circulationaha.120.047164.

10. Inciardi R, Lupi L, Zaccone G, et al. Cardiac involvement in a patient with coronavirus disease 2019 (COVID-19). JAMA Cardiology. 2020; 5(7): 819-824, doi: 10.1001/jamacardio.2020.1096.

11. Kazi DS, Martin LM, Litmanovich D, et al. Case 18-2020: A 73-year-old man with hypoxemic respiratory failure and cardiac dysfunction. N Engl J Med. 2020; 382(24): 2354-2364, doi: 10.1056/NEJMcpc2002417, indexed in Pubmed: 32521138.

12. Newton-Cheh C, Zlotoff DA, Hung J, et al. Case 24-2020: A 44-year-old woman with chest pain, dyspnea, and shock. N Engl J Med. 2020; 383(5) 475-484, doi: 10.1056/NEJMcpc2004975, indexed in Pubmed: 32668103. 
13. Guo T, Fan Y, Chen M, et al. Cardiovascular implications of fatal outcomes of patients with coronavirus disease 2019 (COVID-19). JAMA Cardiology. 2020; 5(7): 811-818, doi: 10.1001/jamacardio.2020.1017.

14. Stone JR, Tran KM, Conklin J, et al. Case 23-2020: A 76-year-old woman who died from Covid-19. N Engl J Med. 2020; 383(4): 380-387, doi: 10.1056/NEJMcpc2004974, indexed in Pubmed: 32640127

15. Ho JSy, Tambyah PA, Ho AFw, et al. Effect of coronavirus infection on the human heart: A scoping review. Eur J Prev Cardiol. 2020; 27(11): 1136-1148, doi: 10.1177/2047487320925965, indexed in Pubmed 32423250 .

16. Leone $\mathrm{O}$, Pieroni $M$, Rapezzi $\mathrm{C}$, et al. The spectrum of myocarditis: from pathology to the clinics. Virchows Arch. 2019; 475(3) 279-301, doi: 10.1007/s00428-019-02615-8, indexed in Pubmed 31297595

17. Ho JSy, Sia CH, Chan MYy, et al. Coronavirus-induced myocarditis: A meta-summary of cases. Heart Lung. 2020; 49(6): 681-685, doi 10.1016/j.hrtlng.2020.08.013, indexed in Pubmed: 32861884

18. Coronavirus-induced myocarditis: A meta-summary of cases. https://www.ncbi.nlm.nih.gov/pmc/articles/PMC7440036/ (Mar. 14 2021)

19. Shi S, Qin Mu, Shen Bo, et al. Association of cardiac injury with mortality in hospitalized patients with COVID-19 in Wuhan, China. JAMA Cardiol. 2020; 5(7): 802-810, doi: 10.1001/jamacardio.2020.0950, indexed in Pubmed: 32211816

20. Zhou F, Yu T, Du R, et al. Clinical course and risk factors for mortality of adult inpatients with COVID-19 in Wuhan, China: a retrospective cohor study. The Lancet. 2020; 395(10229): 1054-1062, doi: 10.1016/s01406736(20)30566-3.

21. Zou X, Chen Ke, Zou J, et al. Single-cell RNA-seq data analysis on the receptor ACE2 expression reveals the potential risk of different human organs vulnerable to 2019-nCoV infection. Front Med. 2020 14(2): 185-192, doi: 10.1007/s11684-020-0754-0, indexed in Pubmed 32170560 .

22. Hikmet $F$, Méar $L$, Edvinsson $\AA$, et al. The protein expression profile of ACE2 in human tissues. Mol Syst Biol. 2020; 16(7): e9610, doi: 10.15252/msb.20209610, indexed in Pubmed: 32715618

23. Rahman $N$, Basharat $Z$, Yousuf $M$, et al. Virtual screening of natura products against Type II Transmembrane Serine Protease (TMPRSS2) the Priming Agent of Coronavirus 2 (SARS-CoV-2) . Molecules. 2020 25(10), doi: 10.3390/molecules25102271.

24. Topol EJ. COVID-19 can affect the heart. Science. 2020; 370(6515) 408-409, doi: 10.1126/science.abe2813, indexed in Pubmed: 32967937

25. Bansal M. Cardiovascular disease and COVID-19. Diabetes Metab Syndr. 2020; 14(3): 247-250, doi: 10.1016/j.dsx.2020.03.013, indexed in Pubmed: 32247212
26. Akhmerov A, Marbán E. COVID-19 and the heart. Circ Res. 2020; 126(10): 1443-1455, doi: 10.1161/CIRCRESAHA.120.317055, indexed in Pubmed: 32252591

27. Bailey AL, Dmytrenko O, Greenberg L, et al. SARS-CoV-2 infects human engineered heart tissues and models COVID-19 myocarditis. JACC Basic Transl Sci. 2021; 6(4): 331-345, doi: 10.1016/j. jacbts.2021.01.002, indexed in Pubmed: 33681537.

28. Bojkova D, Wagner JUG Shumliakivska M et al. SARS-CoV-2 infects and induces cytotoxic effects in human cardiomyocytes. Cardiovasc Res. 2020; 116(14): 2207-2215, doi: 10.1093/cvr/cvaa267, indexed in Pubmed: 32966582

29. Sharma A, Garcia G, Arumugaswami V, et al. Human iPSC-derived cardiomyocytes are susceptible to SARS-CoV-2 infection. Cell Rep Med. 2020; 1(4), doi: 10.1101/2020.04.21.051912, indexed in Pubmed: 32835305

30. Wenzel P, Kopp S, Göbel S, et al. Evidence of SARS-CoV-2 mRNA in endomyocardial biopsies of patients with clinically suspected myocarditis tested negative for COVID-19 in nasopharyngeal swab. Cardiovasc Res. 2020; 116(10): 1661-1663, doi: 10.1093/cvr/cvaa160, indexed in Pubmed: 32562489.

31. Escher F, Pietsch H, Aleshcheva G, et al. Detection of viral SARS-CoV-2 genomes and histopathological changes in endomyocardial biopsies. ESC Heart Fail. 2020; 7(5): 2440-2447, doi: 10.1002/ehf2.12805, indexed in Pubmed: 32529795.

32. Tavazzi G, Pellegrini C, Maurelli M, et al. Myocardial localization of coronavirus in COVID-19 cardiogenic shock. Eur J Heart Fail. 2020; 22(5): 911-915, doi: 10.1002/ejhf.1828, indexed in Pubmed: 32275347.

33. Varga Z, Flammer $A$, Steiger $P$, et al. Endothelial cell infection and endotheliitis in COVID-19. The Lancet. 2020; 395(10234): 1417-1418, doi: 10.1016/s0140-6736(20)30937-5

34. Lindner D, Fitzek $A$, Bräuninger $\mathrm{H}$, et al. Association of cardiac infection with SARS-CoV-2 in confirmed COVID-19 autopsy cases. JAMA Cardiol. 2020; 5(11): 1281-1285, doi: 10.1001/jamacardio.2020.3551, indexed in Pubmed: 32730555.

35. Pesaresi M, Pirani F, Tagliabracci A, et al. SARS-CoV-2 identification in lungs, heart and kidney specimens by transmission and scanning electron microscopy. Eur Rev Med Pharmacol Sci. 2020; 24(9): 5186-5188, doi: 10.26355/eurrev 202005 21217, indexed in Pubmed: 32432787.

36. Bradley B, Maioli H, Johnston R, et al. Histopathology and ultrastructural findings of fatal COVID-19 infections in Washington State: a case series. The Lancet. 2020; 396(10247): 320-332, doi: 10.1016/s01406736(20)31305-2.

37. Bulfamante GP, Perrucci GL, Falleni M, et al. Evidence of SARS-CoV-2 transcriptional activity in cardiomyocytes of COVID-19 patients with outclinical signs of cardiac involvement. Biomedicines. 2020; 8(12), doi: 10.3390/biomedicines8120626, indexed in Pubmed: 33352880. 